\title{
Demonstration of Light-Matter Micro-Macro Quantum Correlations
}

\author{
Alexey Tiranov, ${ }^{1}$ Jonathan Lavoie, ${ }^{1}$ Peter C. Strassmann, ${ }^{1}$ Nicolas Sangouard, ${ }^{2}$ Mikael Afzelius, ${ }^{1}$ \\ Félix Bussières, ${ }^{1}$ and Nicolas Gisin ${ }^{1}$ \\ ${ }^{1}$ Group of Applied Physics, University of Geneva, CH-1211 Geneva 4, Switzerland \\ ${ }^{2}$ Department of Physics, University of Basel, CH-4056 Basel, Switzerland
}

(Received 14 October 2015; published 11 May 2016)

\begin{abstract}
Quantum mechanics predicts microscopic phenomena with undeniable success. Nevertheless, current theoretical and experimental efforts still do not yield conclusive evidence that there is or is not a fundamental limitation on the possibility to observe quantum phenomena at the macroscopic scale. This question prompted several experimental efforts producing quantum superpositions of large quantum states in light or matter. We report on the observation of quantum correlations, revealed using an entanglement witness, between a single photon and an atomic ensemble of billions of ions frozen in a crystal. The matter part of the state involves the superposition of two macroscopically distinguishable solid-state components composed of several tens of atomic excitations. Assuming the insignificance of the time ordering our experiment indirectly shows lightmatter micro-macro entanglement. Our approach leverages from quantum memory techniques and could be used in other systems to expand the size of quantum superpositions in matter.
\end{abstract}

DOI: 10.1103/PhysRevLett.116.190502

Quantum mechanics has been tested in many situations with a remarkably excellent agreement between theory and experiments. There remains, however, one interesting challenge, namely, to demonstrate quantum effects at larger and larger scales [1-3]. This is a timely topic, especially with the advance of quantum technologies that allow one to entangle many kinds of systems involving photons, artificial solid-state atoms, trapped ions, atomic ensembles, nanomechanical oscillators, and large molecules, to name but a few. These approaches all involve "individual" quantum systems (even though each system may be composed of a large number of particles) and should be distinguished from ensemble quantum effects such as superconductivity [4]. These individual systems offer a unique approach to study macroscopic quantum effects, which raises interesting questions: How far can entanglement hold in such systems? How can one compare different systems?

There are many approaches trying to define what constitutes a quantum superposition of macroscopic states [5-7]. The one we use is based on the distinguishability between the states forming the superposition, as formalized in Ref. [8]. More precisely, we say that two quantum states are macroscopically distinct if they can be distinguished with a detector that has a coarse-grained resolution, and we use "macroscopic" to mean "macroscopically distinguishable." This introduces some degree of arbitrariness in what should be the minimum level of coarse-graining, which reflects the challenge of defining such a measure. Instead of trying to achieve this, we use a way to compare different kinds of states to assign them an effective size, as detailed in Ref. [9]. Consequently, the number of particles (or photons) is not used to define the macroscopic nature of the superposition state. Rather, the number of particle is a property of the state that, when increasing, makes the two components easier to distinguish with a given coarsegrained detector (and hence look more like distinct macroscopic objects). Interestingly, the more distinguishable the states become, the more challenging it is to experimentally reveal that they have quantum features (such as entanglement in a micro-macro entangled state) [8], which explains why we do not easily observe such kind of states.

Quantum optics offers a powerful approach to study the quantum features of superpositions of macroscopic states. Purely photonic experiments for example have reported on superposition of coherent states with opposite phases [10-14], squeezing [15,16], and micro-macro entanglement [17-21]. Hybrid systems have also been exploited for micro-macro entanglement where the micro part was an atom and the macro part contained up to 4 photons [22]. It was proposed to use mirror-Bose-Einstein condensate to observe macroscopic quantum superpositions between light and matter [23]. In matter, GHZ-type states have been produced with up to 14 trapped ions [24]. Here we report on the observation of quantum correlations between a single photon and an atomic ensemble containing up to 47 atomic collective excitations. We give evidence that it constitutes genuine light-matter micro-macro entanglement. Hereinafter in the article the term micro-macro entanglement is used assuming the irrelevance of the time order of the measurements. Correctness of this approximation was recently emphasized with a delayed-choice entanglement swapping experiment [25].

Our implementation, inspired from the proposal of Ref. [26] and experiments [18,19], lies within this scenario. More precisely, we start from two photons entangled in polarization and use a local displacement operation to displace, in optical phase space, one polarization mode of 
one photon from the pair. The displacement populates one of the polarization modes with a large number of photons, without affecting the amount of entanglement. The displaced photon is then mapped to an atomic ensemble, creating the light-matter micro-macro entangled state.

Our experiment is conceptually represented on Fig. 1(a). First, an entangled photon pair is generated in the micromicro state

$$
|\psi\rangle=\frac{1}{\sqrt{2}}\left(|1,0\rangle_{s}|1,0\rangle_{i}+|0,1\rangle_{s}|0,1\rangle_{i}\right)
$$

where $s$ and $i$ subscripts are two modes corresponding to the generated signal and idler single photon, while $|1,0\rangle_{s(i)} \equiv|H\rangle_{s(i)}$ and $|0,1\rangle_{s(i)} \equiv|V\rangle_{s(i)}$ correspond to the horizontal polarization state of the signal (idler) photon and the vertical polarization state, respectively. To displace one of the polarization modes of $s$, the signal photon is superimposed with a horizontally polarized coherent state pulse (CSP) on a highly transmissive beam splitter. This corresponds to a unitary displacement operation $\mathcal{D}_{H}^{s}(\alpha)$ on the horizontal mode of the signal photon transmitted through the beam splitter [27]. The average number of photons contained in the displacement pulse is given by $|\alpha|^{2}$. After displacement, the state is written as

$$
|\Psi\rangle=\frac{1}{\sqrt{2}}\left[\left(\mathcal{D}_{H}^{s}(\alpha)|1,0\rangle\right)_{s}|1,0\rangle_{i}+|\alpha, 1\rangle_{s}|0,1\rangle_{i}\right]
$$

This micro-macro entangled state (denoted with a capital $\Psi$ for emphasis) contains a displaced single-photon state of the form $\mathcal{D}(\alpha)|1\rangle$ in the first term, and a coherent state $|\alpha\rangle=\mathcal{D}(\alpha)|0\rangle$ in the second. The idler photon plays the role of the "micro" component of the entangled state. Importantly, increasing $|\alpha|$ makes these two terms become more and more distinguishable when using a coarsegrained detector (on the signal mode) [9]. This is discussed in detail below.

We use a quantum memory (QM) protocol to coherently map the state of the signal mode to the collective state of an ensemble of neodymium atoms frozen in a crystal host [32]. This creates $\eta_{\text {abs }}|\alpha|^{2}$ atomic excitations on average, where $\eta_{\mathrm{abs}}$ is the absorption probability of the QM. The atomic state obtained after this linear mapping contains the atomic equivalents of the optical states $|\alpha\rangle$ and $\mathcal{D}(\alpha)|1\rangle$ [5]. These atomic states can in principle be directly distinguished using a readout technique that has an intrinsically limited microscopic resolution, as it was shown experimentally in Ref. [33]. Instead, here we analyze the reemission and infer, i.e. indirectly, the atomic state from (a)

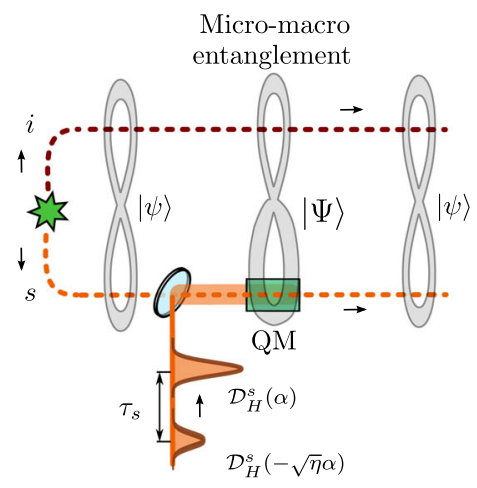

(b)

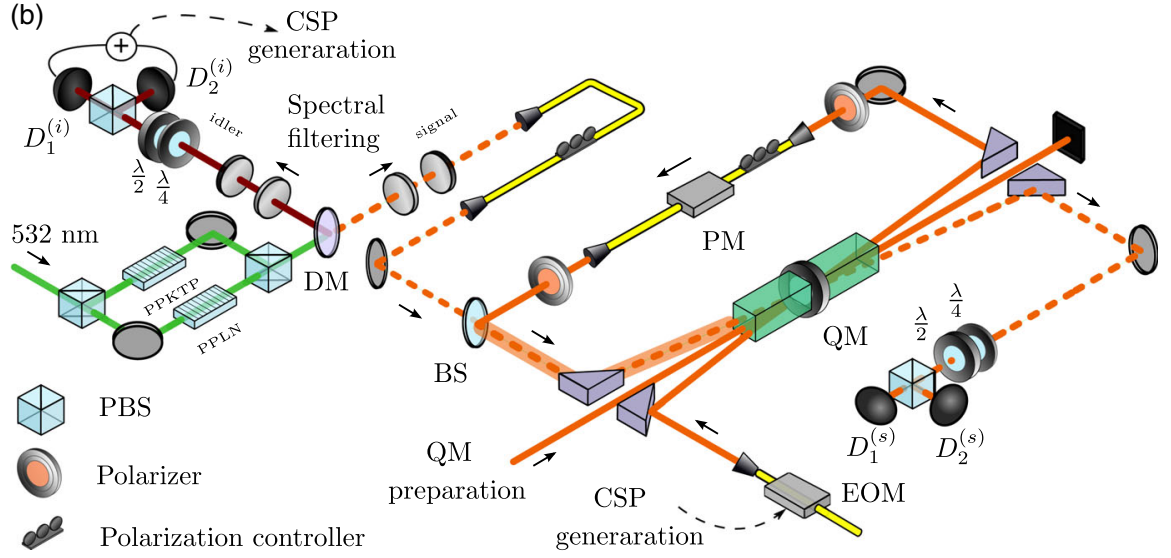

FIG. 1. Experimental scheme. (a) Conceptual scheme for the creation and analysis of the light-matter micro-macro entangled state $|\Psi\rangle$. First, a displacement operation $\mathcal{D}_{H}^{s}(\alpha)$ is applied on the signal mode of the micro-micro polarization entangled state $|\psi\rangle$ using a beam splitter (BS) with high transmittance. The displaced signal photon of the micro-macro state $|\Psi\rangle$ is then mapped inside a solid-state quantum memory $(\mathrm{QM})$ that has a storage and retrieval efficiency $\eta$. To characterize the state, it is first displaced back to $|\psi\rangle$ (in the ideal case) when it is retrieved from the memory using $\mathcal{D}_{H}^{s}(-\sqrt{\eta} \alpha)$, and is then analyzed using various entanglement witnesses. (b) Detailed setup. A polarization entangled pair of photons is created using spontaneous parametric down-conversion from two periodically poled nonlinear waveguides (PPLN and PPKTP) placed in the arms of a polarization interferometer [28] seeded by a continuous wave laser (532 nm wavelength). Dichroic mirror (DM) is used to separate two photons spatially. After the spectral filtering the idler photon is detected by one of the detectors $\left(D_{1}^{(i)}\right.$ or $\left.D_{2}^{(i)}\right)$. This event heralds a single photon in the signal mode, and it triggers the generation of a coherent state pulse (CSP) using an electro-optical intensity modulator (EOM) that carves a pulse out of a continuous wave laser at $883 \mathrm{~nm}$. The CSP is sent in the QM in a different spatial mode than the signal mode. This further allows preparing both the displacement and back-displacement pulses with the required delay and amplitudes (see text and [29] for details). The relative phase necessary for this is set by an electro-optic phase modulator (PM). The first displacement pulse $\mathcal{D}_{H}^{s}(\alpha)$ is synchronized with the heralded single photon on a BS that has a $99.5 \%$ transmittance. The resulting state $|\Psi\rangle$ is stored inside the QM and released after a predetermined time of $\tau_{s}=50 \mathrm{~ns}$. The second displacement $\mathcal{D}_{H}^{s}(-\sqrt{\eta} \alpha)$ is then applied on the state retrieved from the QM. The state is analyzed, together with the idler photon previously measured, using free-space polarization analyzers composed of quarter-wave $(\lambda / 4)$ and half-wave $(\lambda / 2)$ plates followed by polarizing beam splitters (PBS). 
a model using independent measurements discussed in the text. Thus, after a predetermined storage time $\tau_{s}=50 \mathrm{~ns}$, the atomic state is mapped back to the optical signal mode. We note that the storage time is much shorter than the $57 \mu \mathrm{s}$ coherence time and $300 \mu$ s lifetime of the optical transition. Hence, the collective atomic state is coherent throughout the whole process.

As part of the measurement of the light-matter entangled state, the state retrieved from the QM is first displaced back with $\mathcal{D}_{H}^{s}(-\sqrt{\eta} \alpha)$, where the amplitude is reduced by $\sqrt{\eta}$ to match the limited storage efficiency $\eta$ of the QM. To achieve this, an optical pulse is sent through the QM. The timing is such that the part of this pulse that is transmitted (i.e. not absorbed) by the QM precisely overlaps with the displaced signal photon retrieved from the QM. This is equivalent to overlapping them on a beam splitter that has a limited transmittance, and thus it corresponds to a displacement operation accompanied by loss (see [29] for details). In the ideal case, the back-displacement would entirely remove the initial displacement and yield the original micro-micro optical entangled state $|\psi\rangle$. In practice, the displacement back is never perfect in amplitude and phase, which creates noise that limits the maximum size of macroscopic component that can be observed. We note that the displacement happens after the detection of the idler photon. This order could be reversed by using a pulsed laser to generate the entangled photons. The formalism of quantum mechanics indicates that this would lead to the same results as the ones observed here (see [29] for details). Under this natural assumption, our results demonstrate light-matter micro-macro entanglement.

As shown in Fig. 1(b) a $532 \mathrm{~nm}$ continuous wave laser is coherently pumping two nonlinear waveguides, which probabilistically creates photon pairs at $883 \mathrm{~nm}$ (the signal photon) and $1338 \mathrm{~nm}$ (the idler photon). Each photon pair is in superposition of being created in the first waveguide (with horizontal polarizations) and in the second waveguide (with vertical polarizations). Recombination of the output modes of the waveguides leads to a state that is close to the maximally entangled state (1) [28]. The spectrum of the idler photon (the signal photon) is filtered to a Lorentzian linewidth FWHM of $240 \mathrm{MHz}(600 \mathrm{MHz})$ using the combination of a Fabry-Perot cavity (etalon) and a highly reflective volume Bragg grating (see Ref. [28] for details). Detection of the idler photon by detector $D_{1}^{(i)}$ or $D_{2}^{(i)}$ heralds a single photon in the signal mode. The detection signal is also used to generate a CSP using an electrooptical intensity modulator which carves a pulse out of a continuous wave laser at $883 \mathrm{~nm}$.

The QM is based on the atomic frequency comb storage protocol [32]. To store light with an arbitrary polarization, we use a configuration consisting of two inline neodymiumdoped yttrium orthosilicate crystals $\mathrm{Nd}^{3+}: \mathrm{Y}_{2} \mathrm{SiO}_{5}$ separated by a half-wave plate. This configuration was previously used to faithfully store polarization qubits [34-36], to perform light-to-matter quantum teleportation [37] and to store hyperentanglement [38]. The bandwidth of the prepared QM is $600 \mathrm{MHz}$ and it stores photons for $50 \mathrm{~ns}$ with an overall efficiency of $\eta=4.6(2) \%$. The back-displacement operation is performed with an interference visibility of $99.85 \%$, which is remarkably close to being perfect; this is crucial to maximize the size of the displacement.

To quantify how much of the light contained in the displacement pulse is actually displacing the single photon, we must evaluate to what extent their modes are indistinguishable [9]. This was done using Hong-Ou-Mandel interference and measured a visibility of $74 \%$ compared to $85 \%$ expected (see [29] for details).

To reveal quantum correlations in the light-matter micromacro state, we use two methods: the violation of a Clauser-Horne-Shimony-Holt (CHSH) Bell inequality [39] and quantum state tomography.

We first performed the CHSH test without any displacement operations and obtained a parameter $S=2.59(3)$, which is above the local bound of 2 by 20 standard deviations. This was then repeated with an increasing displacement size $|\alpha|^{2}$. The results shown on Fig. 2 are in a good agreement with a theoretical model based on independently measured experimental parameters (see [29] for details). We note that the bases used for all CHSH tests are composed of states of even superposition of $|H\rangle$ and $|V\rangle$. A value of $S=2.099(31)$ is obtained for a displacement containing a mean photon number of $|\alpha|^{2}=13.3(3)$ before mapping the state in the QM. Using the absorption probability $\eta_{\mathrm{abs}} \approx 55 \%$, this corresponds to about 7 excited atoms (see [29] for details). Interestingly, violating the

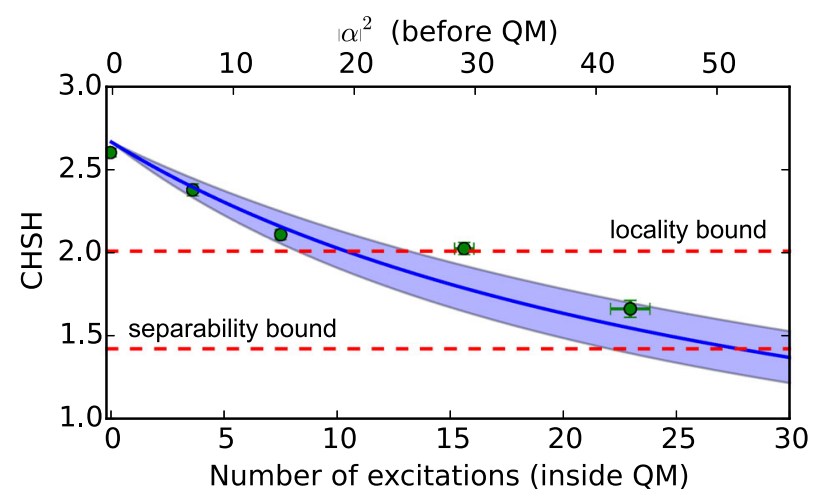

FIG. 2. Measured values of the $S$ parameter of the CHSH-Bell inequality (dots) as a function of the size of the displacement before the QM (top $x$ axis) or as a function of the average number of atomic excitations inside the QM (bottom $x$ axis). $\mathrm{CHSH}$ violation values are above the local bound with up to 7 excitations on average, and above the entanglement bound with up to 23 excitations on average. The error bars are estimated assuming Poisson statistics for the detections. The solid line is obtained from a theoretical model based on independently measured parameters, and the shaded area represents a one standarddeviation uncertainty on the predictions of the model. 
CHSH inequality shows that the light-matter micro-macro state could lead to the strongest form of quantum correlations, namely, nonlocal correlations. Alternatively, the Bell inequality can be used as an entanglement witness if we find $S \geq \sqrt{2} \approx 1.41$ [40]. We measured $S=1.65(5)$ with a mean photon number of $|\alpha|^{2}=42(2)$ before the QM, corresponding to $\approx 23$ excited atoms inside the QM. This is above the separability bound by 4 standard deviations.

To fully characterize the entanglement of the retrieved micro-micro quantum state, we performed an overcomplete set of tomographic measurements and reconstructed the full density matrix. To prove that the state is still entangled we use two criteria, namely, the positivity under partial transposition (PPT) [41], and the concurrence (which is based on the concept of the entanglement of formation) [42]. Figure 3(a) shows results obtained for increasing the size of the displacement. A negative value of $-0.055(10)$ is obtained for the PPT test and a positive concurrence of $0.246(41)$ is obtained for displacements with $|\alpha|^{2}=86(3)$ photons before the QM. This corresponds to $\approx 47$ excited atoms in the atomic ensemble. These results are in a good agreement with our theoretical model described in the SM. We attribute the scatter of the data to the fluctuations of the visibility of the back-displacement operation.

The reported light-matter state can be considered as a micro-macro entangled state for the following reason. Let us illustrate first how the size of a given state can be evaluated from the coarse-grained measure presented in Ref. [9] by focusing on the state (2), which can be rewritten as

$$
\begin{aligned}
& {\left[\mathcal{D}_{H}^{s}(\alpha)\left(|0\rangle_{s H}+|1\rangle_{s H}\right)\right]\left(|0\rangle_{s V}|1,0\rangle_{i}+|1\rangle_{s V}|0,1\rangle_{i}\right)} \\
& \quad-\left[\mathcal{D}_{H}^{s}(\alpha)\left(|0\rangle_{s H}-|1\rangle_{s H}\right)\right]\left(|0\rangle_{s V}|1,0\rangle_{i}-|1\rangle_{s V}|0,1\rangle_{i}\right),
\end{aligned}
$$

where the normalization is omitted. The state therefore involves the superposition of $\mathcal{D}_{H}^{s}(\alpha)(|0\rangle+|1\rangle)$ and $\mathcal{D}_{H}^{s}(\alpha)(|0\rangle-|1\rangle)$ in the horizontal mode of the signal photon, and one can obtain one or the other by measuring the idler photon in the basis of diagonal polarizations. Although these two components partially overlap in the photon number space, the distance between their mean photon numbers is given by $2|\alpha|$; see Fig. 3(b). For $|\alpha|^{2} \gtrsim 2$, they can be distinguished with a single measurement with a probability of $\approx 91 \%$ using a detector that has a perfect resolution [9]. If measured with a coarse-grained detector, this probability is reduced to $50 \%$ when the coarse graining is of the order of $|\alpha|$ or more. The effective size of the state (2) can be naturally quantified by the maximum coarse-graining $\sigma_{\max }$ that allows one to distinguish the two components $\mathcal{D}_{H}^{s}(\alpha)(|0\rangle+|1\rangle)$ and $\mathcal{D}_{H}^{s}(\alpha)(|0\rangle-|1\rangle)$ with a given probability $P_{g}$, where $P_{g}$ should be significantly above $50 \%$ to be meaningful for a single-shot measurement. Similarly, the effective size can be evaluated by comparing the results to an archetypical state involving the superposition of $|0\rangle$ and $|N\rangle$ Fock states, where $N$ is the smallest

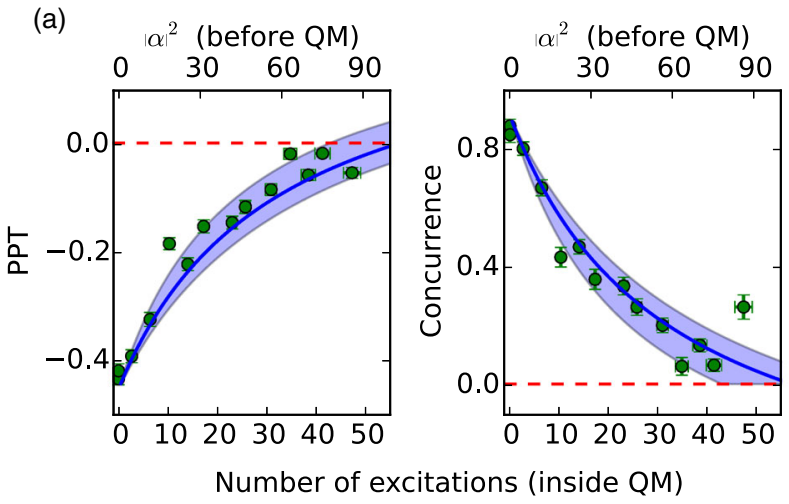

(b)

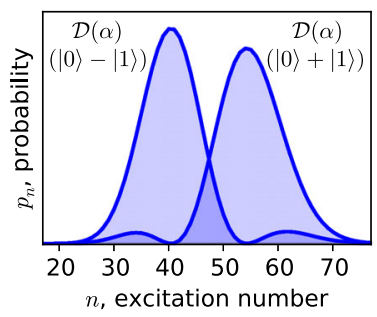

FIG. 3. Quantum state characterization. (a) PPT and concurrence values (obtained from quantum state tomography) as a function of the size of the displacement before the QM (top $x$ axis) or as a function of the average number of atomic excitations inside the QM (bottom $x$ axis). The error bars are estimated from Monte Carlo simulations assuming Poisson noise. The PPT criteria remains negative and the concurrence value remains positive with up to 47 excitations on average. The solid lines in all graphs are obtained from a theoretical model based on the independently measured parameters, and the shaded areas are the uncertainty on these parameters. (b) Distribution of number of atomic excitations of the two macroscopically distinguishable components $\mathcal{D}(\alpha)|1\rangle$ and $|\alpha\rangle$ when expressed in the $\{|0\rangle+|1\rangle,|0\rangle-|1\rangle\}$ basis.

value that allows distinguishing $|0\rangle$ from $|N\rangle$ with a probability $P_{g}$ and a coarse graining $\sigma_{\max }$. From our results, which are well reproduced by our theoretical model based on independent measurements, we can confidently give an estimate of the size of the light-matter state from which the entanglement is measured. For $P_{g}=2 / 3$, the state is analogous to the state $|\uparrow\rangle|0\rangle+|\downarrow\rangle|N\rangle$ with $N \approx 13$, where $|\uparrow\rangle$ and $|\downarrow\rangle$ represent microscopic orthonormal states.

Naturally, one must also carefully consider the effect of loss in the signal mode before the beam splitter used for the displacement, as well as the absorption probability in the QM. In the SM we show that if the heralding probability to find the signal photon at the beam splitter is $\eta_{h}$ and the absorption in the QM is $\eta_{\text {abs }}$, the displacement creates a mixture of the state $|\Psi\rangle$ with a displacement of amplitude $\sqrt{\eta_{\mathrm{abs}}} \alpha$ with probability $\eta_{h} \eta_{\mathrm{abs}}$, and a separable state with the complimentary probability. In our case we have $\eta_{h} \eta_{\mathrm{abs}} \approx 10 \%$, which makes the two macroscopic states nearly indistinguishable, even with a detector with perfect microscopic resolution. This exemplifies that the direct observation of macroscopic features is a very challenging task. Nevertheless, we stress that 
the entanglement signature that we directly observe is stemming from the micro-macro state component of the mixture, whose effective size is defined as above. The observation of this entanglement, and its behavior with increasing size, is the main result of this Letter. The direct observation of the size of the superposition with an actual coarse-grained detector is left for future work. This would require reduced loss and a highly efficient quantum memory. Achieving this is certainly conceivable, given the large storage efficiencies that can now be obtained with some quantum memories [43] and with the progress of linear detectors to achieve subshot-noise resolution (e.g. see [44]). Homodyne detection could also prove useful for distinguishing the states, as demonstrated in Ref. [19]. Overall, our approach could certainly be improved with other types of quantum memory, which has the potential to yield larger quantum superpositions in matter.

We thank Florian Fröwis, Jean Etesse, Anthony Martin, Natalia Bruno and Pavel Sekatski for useful discussions, as well as Claudio Barreiro and Raphaël Houlmann for technical support. We thank Harald Herrmann and Christine Silberhorn for lending the PPLN waveguide. This work was financially supported by the European Research Council (ERC-AG MEC) and the National Swiss Science Foundation (SNSF) (including Grant No. PP00P2-150579). J. L. was supported by the Natural Sciences and Engineering Research Council of Canada (NSERC).

[1] M. Brune, E. Hagley, J. Dreyer, X. Maître, A. Maali, C. Wunderlich, J. M. Raimond, and S. Haroche, Phys. Rev. Lett. 77, 4887 (1996).

[2] D. Leibfried, E. Knill, S. Seidelin, J. Britton, R. B. Blakestad, J. Chiaverini, D. B. Hume, W. M. Itano, J. D. Jost, C. Langer, R. Ozeri, R. Reichle, and D. J. Wineland, Nature (London) 438, 639 (2005).

[3] M. Arndt and K. Hornberger, Nat. Phys. 10, 271 (2014).

[4] A. J. Leggett, Prog. Theor. Phys. Suppl. 69, 80 (1980).

[5] F. Fröwis, N. Sangouard, and N. Gisin, Opt. Commun. 337, 2 (2015).

[6] H. Jeong, M. Kang, and H. Kwon, Opt. Commun. 337, 12 (2015).

[7] T. Farrow and V. Vedral, Opt. Commun. 337, 22 (2015).

[8] P. Sekatski, N. Gisin, and N. Sangouard, Phys. Rev. Lett. 113, 090403 (2014).

[9] P. Sekatski, N. Sangouard, and N. Gisin, Phys. Rev. A 89, 012116 (2014).

[10] A. Ourjoumtsev, R. Tualle-Brouri, J. Laurat, and P. Grangier, Science 312, 83 (2006).

[11] J. S. Neergaard-Nielsen, B. M. Nielsen, C. Hettich, K. Mølmer, and E. S. Polzik, Phys. Rev. Lett. 97, 083604 (2006).

[12] K. Wakui, H. Takahashi, A. Furusawa, and M. Sasaki, Opt. Express 15, 3568 (2007).

[13] A. Ourjoumtsev, H. Jeong, R. Tualle-Brouri, and P. Grangier, Nature (London) 448, 784 (2007).
[14] B. Vlastakis, G. Kirchmair, Z. Leghtas, S. E. Nigg, L. Frunzio, S. M. Girvin, M. Mirrahimi, M. H. Devoret, and R. J. Schoelkopf, Science 342, 607 (2013).

[15] T. Eberle, V. Händchen, and R. Schnabel, Opt. Express 21, 11546 (2013).

[16] F. A. Beduini, J. A. Zielińska, V. G. Lucivero, Y. A. de Icaza Astiz, and M. W. Mitchell, Phys. Rev. Lett. 114, 120402 (2015).

[17] F. De Martini, F. Sciarrino, and C. Vitelli, Phys. Rev. Lett. 100, 253601 (2008).

[18] N. Bruno, A. Martin, P. Sekatski, N. Sangouard, R. T. Thew, and N. Gisin, Nat. Phys. 9, 545 (2013).

[19] A. I. Lvovsky, R. Ghobadi, A. Chandra, A. S. Prasad, and C. Simon, Nat. Phys. 9, 541 (2013).

[20] H. Jeong, A. Zavatta, M. Kang, S.-W. Lee, L. S. Costanzo, S. Grandi, T. C. Ralph, and M. Bellini, Nat. Photonics 8, 564 (2014)

[21] O. Morin, K. Huang, J. Liu, H. Le Jeannic, C. Fabre, and J. Laurat, Nat. Photonics 8, 570 (2014).

[22] S. Deléglise, I. Dotsenko, C. Sayrin, J. Bernu, M. Brune, J.-M. Raimond, and S. Haroche, Nature (London) 455, 510 (2008).

[23] F. De Martini, F. Sciarrino, C. Vitelli, and F. S. Cataliotti, Phys. Rev. Lett. 104, 050403 (2010).

[24] T. Monz, P. Schindler, J. T. Barreiro, M. Chwalla, D. Nigg, W. A. Coish, M. Harlander, W. Hänsel, M. Hennrich, and R. Blatt, Phys. Rev. Lett. 106, 130506 (2011).

[25] X.-S. Ma, S. Zotter, J. Kofler, R. Ursin, T. Jennewein, Č. Brukner, and A. Zeilinger, Nat. Phys. 8, 479 (2012).

[26] P. Sekatski, N. Sangouard, M. Stobińska, F. Bussières, M. Afzelius, and N. Gisin, Phys. Rev. A 86, 060301 (2012).

[27] M. G. Paris, Phys. Lett. A 217, 78 (1996).

[28] C. Clausen, F. Bussières, A. Tiranov, H. Herrmann, C. Silberhorn, W. Sohler, M. Afzelius, and N. Gisin, New J. Phys. 16, 093058 (2014).

[29] See Supplemental Material at http://link.aps.org/ supplemental/10.1103/PhysRevLett.116.190502, which includes Refs. [30-31].

[30] V. Caprara Vivoli, P. Sekatski, J.-D. Bancal, C. C. W. Lim, B. G. Christensen, A. Martin, R. T. Thew, H. Zbinden, N. Gisin, and N. Sangouard, Phys. Rev. A 91, 012107 (2015).

[31] V. C. Vivoli, P. Sekatski, J.-D. Bancal, C. C. W. Lim, A. Martin, R. T. Thew, H. Zbinden, N. Gisin, and N. Sangouard, New J. Phys. 17, 023023 (2015).

[32] M. Afzelius, C. Simon, H. de Riedmatten, and N. Gisin, Phys. Rev. A 79, 052329 (2009).

[33] S. L. Christensen, J.-B. Béguin, E. Bookjans, H. L. Sørensen, J. H. Müller, J. Appel, and E. S. Polzik, Phys. Rev. A 89, 033801 (2014).

[34] C. Clausen, F. Bussières, M. Afzelius, and N. Gisin, Phys. Rev. Lett. 108, 190503 (2012).

[35] M. Gündoğan, P. M. Ledingham, A. Almasi, M. Cristiani, and H. de Riedmatten, Phys. Rev. Lett. 108, 190504 (2012).

[36] Z.-Q. Zhou, W.-B. Lin, M. Yang, C.-F. Li, and G.-C. Guo, Phys. Rev. Lett. 108, 190505 (2012).

[37] F. Bussières, C. Clausen, A. Tiranov, B. Korzh, V. B. Verma, S. W. Nam, F. Marsili, A. Ferrier, P. Goldner, H. Herrmann, C. Silberhorn, W. Sohler, M. Afzelius, and N. Gisin, Nat. Photonics 8, 775 (2014).

[38] A. Tiranov, J. Lavoie, A. Ferrier, P. Goldner, V. B. Verma, S. W. Nam, R. P. Mirin, A. E. Lita, F. Marsili, H. Herrmann, C. Silberhorn, N. Gisin, M. Afzelius, and F. Bussières, Optica 2, 279 (2015). 
[39] J. F. Clauser, M. A. Horne, A. Shimony, and R. A. Holt, Phys. Rev. Lett. 23, 880 (1969).

[40] S. M. Roy, Phys. Rev. Lett. 94, 010402 (2005).

[41] A. Peres, Phys. Rev. Lett. 77, 1413 (1996).

[42] S. Hill and W. K. Wootters, Phys. Rev. Lett. 78, 5022 (1997).
[43] F. Bussières, N. Sangouard, M. Afzelius, H. de Riedmatten, C. Simon, and W. Tittel, J. Mod. Opt. 60, 1519 (2013).

[44] T. Gerrits, B. Calkins, N. Tomlin, A. E. Lita, A. Migdall, R. Mirin, and S.W. Nam, Opt. Express 20, 23798 (2012). 